\title{
Design Features of the User Interface of the Pipeline Pressure Control Monitoring System
}

\author{
Elena Afonina ${ }^{1[0000-0002-3560-8054]}$, Marina Levaya ${ }^{1[0000-0003-2977-6200]}$, \\ Igor Levyy 2[0000-0003-2169-4761] \\ ${ }^{1}$ Bryansk State Technical University, Bryansk, Russia \\ ${ }^{2}$ ET LLC “Tsirobs”, Moscow, Russia \\ cvetelena@rambler.ru|mlevaya@mail.ru|i-levyy@mail.ru
}

\begin{abstract}
For control and regulation of hydraulic systems, especially, oil pumping stations, automatic pressure control systems (APCS) are used. Using it, the monitoring, controlling, and adjusting the operation of technological equipment, optimization of modes, and other tasks that require direct human participation become possible. The operator (or dispatcher) interacts with the system via a human-machine interface. The monitoring system includes programs for collecting, processing, displaying, and archiving information about the object of observation and control. Well-suited and being put to the right user interface design promotes not only the effective interaction of the operator with the system in normal conditions but also prevents errors and helps to find a solution in a dangerous or emergency swiftly. This paper presents a software package designed for real-time monitoring and regulation of pipeline operation. A system simulation model is provided for the teaching and training of site personnel.
\end{abstract}

Keywords: Automatic Control system, Computer Modeling, Visualization of Work, Design, User Interface.

\section{Introduction}

Automatic pressure control systems are software and technical systems for automatic pressure control at the reception and outlet of oil pumping stations (NPS). Automated systems allow operators (dispatchers) of oil pumping stations to monitor in real-time the change of pressure in the oil pipeline, and in case of emergencies to take appropriate actions [1,3]. Comprehensive monitoring of the state of the main pipelines is carried out to solve a wide range of problems. High-tech complexes are created to prevent fire hazard situations, monitor temperature and pressure in the hydraulic system, determine the safety margin and evaluate the stress-strain state of the pipeline structure in various climatic

Copyright (C) 2020 for this paper by its authors. Use permitted under Creative Commons License Attribution 4.0 International (CC BY 4.0).

* Publication is supported by RFBR grant № 19-07-00844. 
conditions, etc. High-pressure systems transporting hazardous liquids and gases require both continuous and periodic evaluation of the operation. The development and improvement of control systems are constantly in the field of view of both domestic and foreign specialists developing full-scale software complexes covering the maximum number of technological operations. To date, the level of import dependence of Russian companies in terms of software and APCS in the segment of transportation and storage of petroleum products is about $80 \%$ [13]. The development of domestic software for the oil and gas sector in modern conditions becomes relevant and becomes the key to ensuring the safety of the industry $[12,13]$.

The reliability and safety of the NPS depend on many components - high-quality equipment, high-tech software and technical complexes, workplace automation, as well as personnel qualifications. To create and maintain the correct and reliable management skills in regular, emergency and emergencies, the system interface should reflect the essence of regulated processes, clarify the task and make decisions. At the moment, the issue of developing an automated system for monitoring and regulating pressure in the oil pipeline, taking into account the human and intellectual factor of users, is relevant.

The task is to develop a convenient and functional interface of the control complex for effective human-machine interaction, to include a simulation mode of operation.

\section{Setting goals and implementation methods}

In the process of designing the software package, research and solution search were carried out in the following areas:

- $\quad$ selection of the SCADA package taking into account the requirements for the software package;

- $\quad$ use of a mathematical model of oil pipeline pressure control by flow throttling method;

- creation of a control algorithm for the automatic pressure control system taking into account the non-linear nature of the load;

- development of a software system model;

- development of the stand of the visual control of work of a system including the interface, forms and algorithms for work of operators;

- testing and debugging of the system taking into account the inclusion of training and training opportunities for personnel.

Operating conditions in different regions may differ both in the capacity of the NPS, the level of automation of the station infrastructure, the structural features of the equipment in operation, and in the range of tasks that are the responsibility of the operators. Interactive control should not be a source of stress or psychological discomfort for the operator. The screen type should be familiar, that is, take into account the traditional approach to designing the user interface in the industry $[7,9,16]$. The automated system should take into account possible changes in the applied hardware and have, among others, a simulation model for use as a training complex [5, 11].

Currently, SCADA systems (or SCADA technologies), which are multi-functional software packages, are used to develop automated systems for visualizing work and monitoring complex dynamic processes. The main functions of SCADA systems are 
dispatch control, alarm in case of non-standard situations, event logging, documentation and archiving of data $[12,14]$. When analyzing the main groups of indicators (technical characteristics, cost, operability) of several SCADA systems, it was determined that the best is the domestic system TraceMode.

Regulation of liquid flow at oil pumping stations is involved in maintaining the specified parameters at standard, as well as the occurrence of abnormal and emergency situations. Pressure surges can occur as a result of seasonal fluctuations in production, changes in the physical parameters of pumped oil and the appearance of other transient processes in oil pipelines. In some cases, these changes may lead to the shutdown, repair of the NTC and other adverse situations, accompanied by economic losses. The means of regulating the pressure in the hydraulic system, which performs equipment protection and system operation as a whole, is the flow throttling method. The control mechanism is the dampers rotating by a certain angle depending on the pressure value in the pipeline.

The mathematical model for calculating the nonlinear mechanical characteristic of the flow load, as well as the automatic reconfiguration of the pressure regulator, should take into account several parameters: viscosity and density of the medium, geometric characteristics of the shutter (diameter, length, cross-section area), flow rate, average calculated fluid flow rate. When constructing a hydrodynamic flow model, in works devoted to the study of the dependence of the liquid pressure in the oil pipeline on the angle of rotation of the control flap $[3,4,14]$, specific zones with a characteristic flow in the pipeline were identified: a zone of uniform flow, a zone of sharp expansion, a zone of slow constriction, a zone of sharp constriction. Mathematical dependencies that allow calculating the pressure level on the shutter depending on the angle of rotation formed the basis for the construction of the pressure stabilization algorithm $[3,14]$.

The algorithm is designed so that data from the controller enters the system that performs the processing and calculation of parameters. If the pressure value exceeds the permissible limits, the angle of shutter rotation is calculated. The operator can adjust the pressure in the manual mode by setting the rotation angle through the control system interface.

\section{Building a User Interface}

When designing the interface, it was decided to adhere to the following principles.

- Display of control functions shall satisfy standard graphics diagrams $[1,2,16]$. Industry standards for the development of process equipment automation systems specify the types, layout and colour policy of screen forms [16, 17]. The ergonomics of human-machine interaction must be present in the interface elements, without violating the mandatory requirements [9].

- Consistency. The interface should help the operator to develop familiar actions and prevent erroneous ones, therefore, when interacting with elements of the same appearance, the same actions should occur. Each elementary action on the screen must be performed as much as possible in the same way (via menu buttons, or keyboard shortcuts, or response to a request, etc.). The monotonicity of the interface will reduce the time for 
training the operator and will allow you to not waste time in an emergency to think about the usual actions $[8,15]$.

- Flexibility. The use of terms, symbols and graphic information should take into account different skill levels and different intellectual capabilities of the user. This will make it possible to use the system both in real conditions and as a simulator.

- Friendships. The set of commands (questions, symbols) should prevent situations that may end in errors. The opening of dialogue boxes, menu sections, messages that appear should be phased. Therefore, the algorithm for their appearance should provide such an organization of information as is necessary to perform only the next step $[9,10]$.

- Feedback principle. Through the interface, the operator must understand how the system responds to a particular action, feel interaction with the actuators of the entire complex $[11,19]$.

- Taking into account the psychophysiological state of the operator. In addition to the carefully developed visual model of the control object, the level of correspondence of the skills and skills of the operator of the complexity of the task it solves is important. Studies show that if a task is too difficult (with an irrational distribution of functions between a person and a software complex), the operator will be constantly in an alarm state. If the actions are overly simple and monotonous (for example, repeated dialogue boxes, limited capabilities in the decision-making process), attention is reduced, which can lead to erroneous actions [15].

\section{Results}

At the stage of sketch design, several screen reactors were developed. All of them take into account the above principles, as well as some specific requirements for the system. Shape, colour and location of mnemonic signs on the screen are indicators of processes (Fig. 1). The buttons of the upper navigation panel for the requirements of industry standards should be grey and include (as a requirement) the following information: modes, settings, log, diagnostics, date/time $[6,7,16]$.

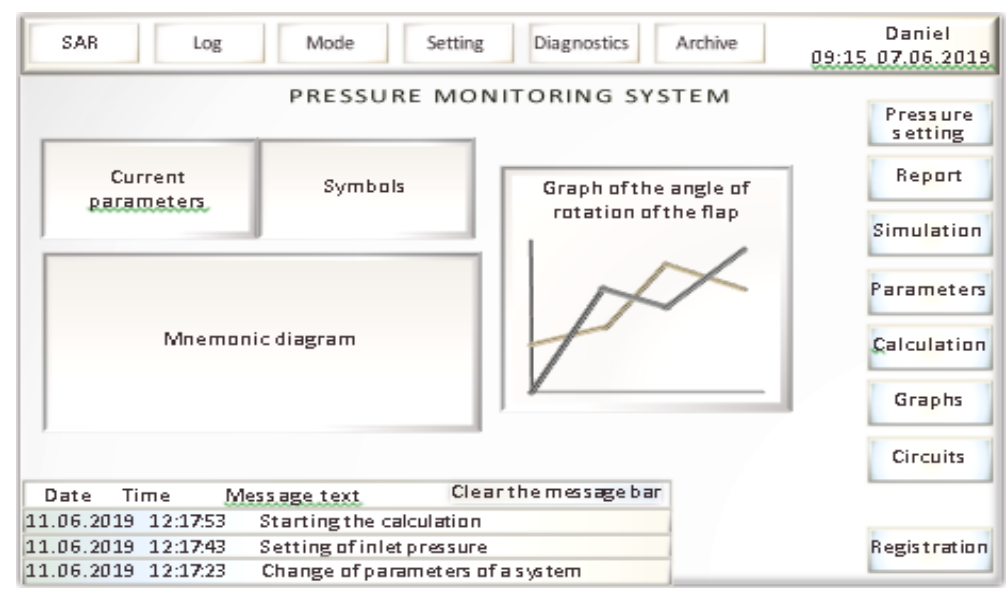

Fig. 1. Diagram of the main window of the pressure monitoring system 
The mnemonic field is a changeable display element. In the centre of the screen, it is proposed to locate the main controlled parameters of the system, built-in real-time graphics, system messages. The operator's constant attention should be located in a certain area of the screen, where the current state of the system will be reflected, as well as ways of acting on it.

The navigation bar displayed at the top of the screen should allow you to display the desired queries. The traditional colours of dispatcher monitors, saturated mainly with steel grey colour, it was decided to soften somewhat, without changing the general colour concept. GOST 14202-69 prescribes the colour of the pipeline for the combustible liquid to be painted brown, and for screen forms the image of the pipeline through which oil and oil products move, the industry-standard recommends painting in black shades [7, 16, 17]. Signal colours remain generally accepted: danger (error or abnormal situation) - red colour, safety or neutrality - green.

You must log in and enter your name and password before you begin (Fig. 2). After completing the authorization (or checking the entered parameters in the database for the registered user), the operator enters the main window of the system, where his name is indicated.

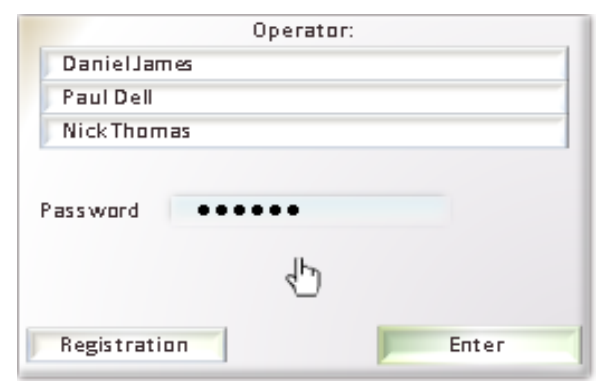

Fig. 2. The registration window of the operator

Multiple screen forms are provided for setting the initial parameters of the system, setting the input and allowable pressure. At the level of interaction, "operator - monitoring system" of the automatic monitoring system, values of measured parameters (pressure, the position of control gates, messages about system events and accidents) are continuously recorded in archive arrays, and trends of monitored parameters are output to the graphical terminal. The interval of trend output, as well as their recording in the archive, is 2 seconds. Archive program is developed for viewing and printing of archive arrays, which allows displaying archival trends and messages for any day, to scale trends, to set time interval for displaying values on the screen, to select measured parameters for display [14].

The user should interact with the system by direct manipulation - working with objects (mnemonic signs or menu buttons) on the screen. Therefore, the appearance of the screen, visual images of tasks and objects require simple and understandable images. Excludes multi-word, complex characters, or graphics. All user data - operator results - must be saved. The dialogue boxes are designed with a save request. 
To view reports, the operator can go to the Report page through the main menu of the system (Fig. 3). The mouse navigates between dialogue boxes and graphs.

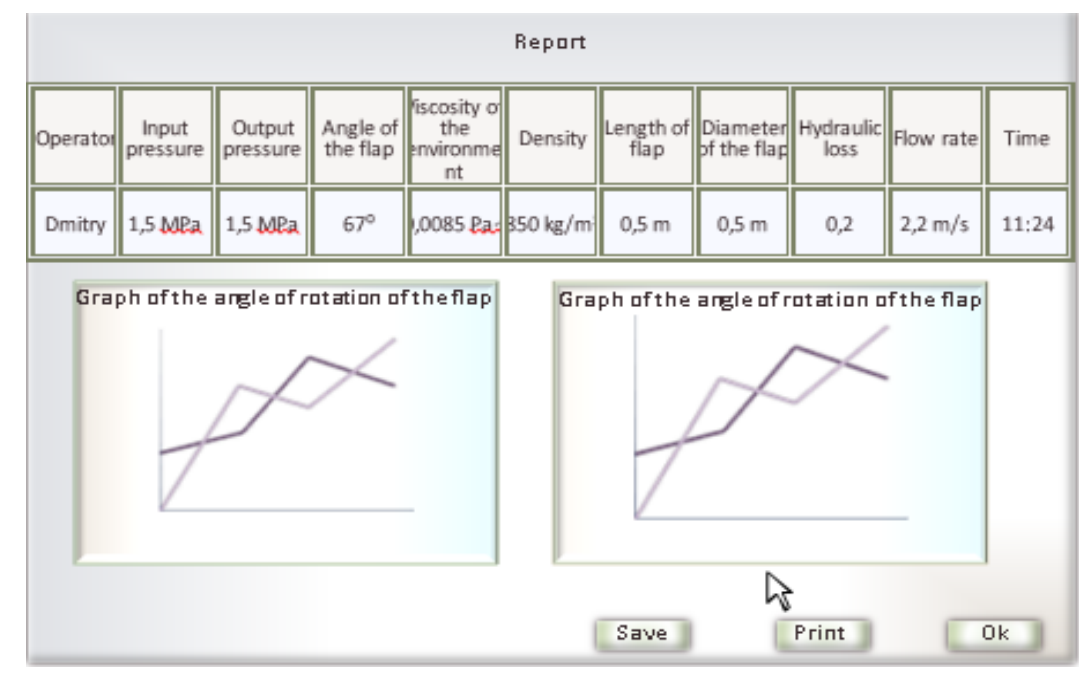

Fig. 3. The report window

To start operation in simulation mode, the system includes the required volume of training functions, as well as the possibility of technical implementation of several scenarios. Not only the rotation angle graph of the adjustment mechanism are displayed, but also graphs showing the change in pressure in the system. This makes it possible to visually interact the operator with the controlled system, which is especially important for the trainee $[19,20]$. When starting the program pressure monitoring system, the user - the operator of the oil refining station - gets to the main window, the main information about the system operation is displayed on the counter (Fig. 4). In the manual documents on one of the screen forms, the location of the pressure control controllers is given. In this interface, only a fragment of this section is given with emphasis on the regulator operating element - shutter. The main window of the system displays graphs of dependencies between the pressure level in the flow and the rotation angle of the furbottom pressure control.

To verify that the product meets the specified requirements, it was necessary to carry out some tests according to the following plan:

- operator registration (system response to input of unregistered user data);

- check of operation with the settings table, data entry;

- construction and display of graphs;

- saving of message history, checking of message log operation;

- checking the creation and preservation of reports, archiving of reports;

- system response to adjustment mechanism position change;

- emergency warning operation; debugging the operator's scenario of operation with the interface in an emergency to eliminate errors in the state of psychological load;

- check of simulation mode operation. 
Design Features of the User Interface of the Pipeline Pressure Control Monitoring System 7

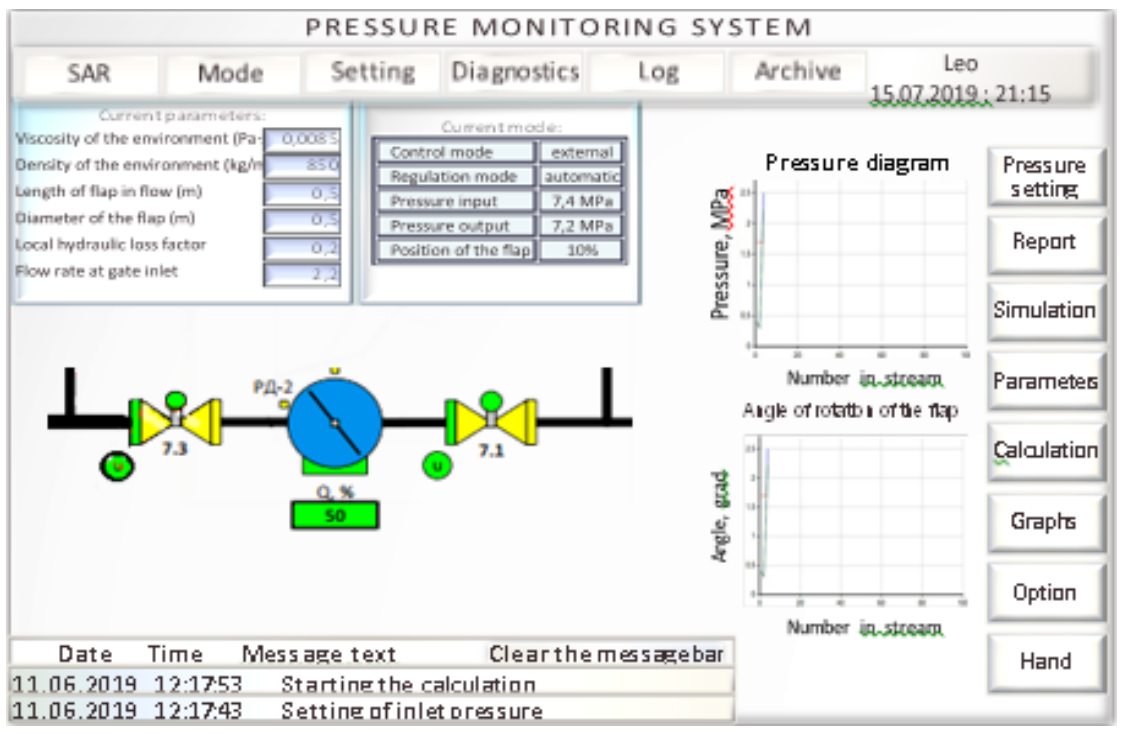

Fig. 4. The main window of the program pressure monitoring system

The main purpose of the testing was to verify the operation of the software, evaluate the ergonomic and functional qualities of the human-machine interface.

\section{Conclusions}

In this work, the original interface of the automatic pressure control system in the pipelines of oil pumping stations was developed. The designed software system is designed for real-time monitoring, collection, processing, sampling and archiving of information about the monitoring and control object. The developed system allows operators to monitor the state of the oil pipeline in real-time and, if necessary, take appropriate actions. In the design of the interface, it was important to combine a traditional approach to designing screen forms of automated workplaces with the usability and visibility of the presented data. Particular attention is paid to visualizing the relationship between the pressure level in the pipeline and the operation of the adjustment mechanism. Special settings of the simulation mode allow you to use this system as a training tool for NTC operators.

\section{References}

1. Automation and telemechanization of the main oil pipelines. Highlights: Guidance Document. RD35 - 240.00-KTH-207-08. PJSC AK Transneft. (2008)

2. Automated process control system of transfer stations: General technical requirements OTT35.240.00-KTH-061-14. PJSC Transneft. (2019).

3. Antropov A.T., Rikkonen S.V. Hydromechanical characteristics of the ECS pressure control flap. i-tech - journal of intelligent technologies. No. 12. Pp. 35-39. (2009) 
4. Bogdanov E.P., Rikkonen S.V. Hydromechanical moment of resistance of the control flap of the main oil pipeline. Izvestiya Tomsk Polytechnic University, Vol. 323, No. 2. pp. 143 146. (2013)

5. Dozortsev, V.M. Computer simulators for training process operators. Synteg. M. (2009)

6. GOST 34182-2017. Main pipeline transport of oil and petroleum products. Operation and maintenance. Basic provisions. Moscow: Standardinform. (2017).

7. GOST 14202-69. Pipe-lines of industrial plants. Identifikation colouring, safety signs and marking screens.

8. GOST RV 29.04.002-84. SEST and EO. Algorithm and structure of operator activity. General ergonomic requirements.

9. GOST R ISO 9241-161-2016. Ergonomics of human-system interaction. Part 161. Elements of the graphical user interface. M.: Standardinform, (2016)

10. Zakharova, A.A., Shklyar A.V. Visual models. Problems of informatics. No. 4. Pp. 41-47. (2010)

11. Ivlev P. Operator panel as a means of creating a highly efficient HMI / Control Engineering Russia. \# 2 (74). (2018). R. 41-46.

12. Ilyinykh T. Future of Automation: HoneyWell Users Group 2019 User Conference for EMEA / Control Engineering Russia. \# 5 (83). (2019). Pp. 12-15.

13. Kozlova D., Pigarev D. Digital oil production: tuning for the industry / Vygon Consulting. (2018).

14. Korostelev D.A., Levaya M.N., Levyy I.S. Automation of the process of monitoring and regulation of pressure in the oil pipeline. Bulletin of AGTU. Ser.: Management, computing and computer science. No. 1. (2017).

15. Nazarenko N. A., Paderno P. I. Influence of the interface on the state and health of the operator / Biotechnosphere. (2009). № 6 (6). Pp. 45-52.

16. Process equipment automation systems. Screen forms: Guidance document 35.240.50 KTN 243 19. PJSC Transneft. (2019)

17. Automatic control system: General technical requirements. OTT-35.240.50-KTH-132-15. PJSC Transneft. (2019)

18. Zacharova A.A., Krysko A., Vekhter E., Shklyar A. Meta-design of processes based on visualization tools/ Communications in computer and information science. 2019. Volume 1083. Pp. 245-255.

19. Zacharova A.A., Vekhter E., Shklyar A.V., Pak A.J. Visual modeling in an analysis of multidimensional data. Jornal of phisics: Conference series. XI international scientific and technical conference "Applied mechanics and dynamics systems". (2018). P. 012127.

20. Weatherford. LOWISTM Life of Well Information Software. http://www.ep-solutions.com/solutions/Software/LOWIS.htm 